\title{
Relationships between a roller and a dynamic pressure distribution in circular hydraulic jumps
}

\author{
Kensuke Yokoi ${ }^{1,2}$ and Feng Xiao ${ }^{3}$ \\ ${ }^{1}$ Division of Mathematics and Research Institute for Electronic Science, Hokkaido University, Sapporo 060-0812, Japan \\ ${ }^{2}$ Computational Science Division, RIKEN (The Institute of Physical and Chemical Research), Wako 351-0198, Japan \\ ${ }^{3}$ Department of Energy Sciences, Tokyo Institute of Technology, Yokohama 226-8502, Japan
}

(To appear in Phys. Rev. E, Vol.61, Feb. 2000)

We investigated numerically the relation between a roller and the pressure distribution to clarify the dynamics of the roller in circular hydraulic jumps. We found that a roller which characterizes a type II jump is associated with two high pressure regions after the jump, while a type I jump (without the roller) is associated with only one high pressure region. Our numerical results show that building up an appropriate pressure field is essential for a roller.

PACS numbers: 83.50.Lh, 47.15.Cb, 47.32.Ff, 83.20.Jp

As can be easily observed in a kitchen sink, a circular hydraulic jump is formed when a vertical liquid jet impinges on a horizontal surface. The schematic figure of the circular hydraulic jump can be shown as in Fig. 1. The phenomenon has been investigated by many researchers through various approaches [111].

In some experiments, the depth on the outside of the jump can be controlled by varying the height of a circular wall $d$, as shown in Fig. 1. Experimental results show that a circular hydraulic jump has two kinds of steady states which can be reached by changing $d[7]$. When $d$ is small or 0, a type I jump is formed, as shown in Fig. 1(a). On increasing $d$ the jump becomes steeper until a critical $d_{c}$ is reached. If $d$ becomes larger than $d_{c}$, the liquid outside of the jump topples. Then another steady state, a type II jump, is formed as shown in Fig. 1(b). The eddy on the surface in a type II jump, a secondary circulation, is usually called a "roller." The existence of a roller distinguishes the two types of jumps.

The roller is a common and important feature for many hydraulic phenomena. Recent experiments [9, 10] demonstrate that various regular polygonal jumps can develop from a circular jump by controlling the height of the outer circular wall, and that all those polygonal jumps are associated with rollers. Rollers are also observed in the channel flows and are useful for dissipating the excess energy of high velocity flows, such as from sluice gates and spillways [3]. It is widely recognized that rollers play an important role in hydraulic engineering.

However, theoretical studies concerning the formation and evolution of the roller in a hydraulic jump are limited because of the largely deformed interface. Some theoretical studies have been proposed using a hydrostatic assumption in the vertical direction. Some reasonable results have been obtained for the flows of type I jumps [8].
However, a type II jump appears to be beyond the regime that this vertical-assumption theoretical model is able to deal with. Numerical modeling has also been used to investigate the circular hydraulic jump problem. Due to the difficulties in the numerical treatment of largely distorted interfacial flows, the free boundary of the liquid surface was treated as the fixed boundary of a prescribed shape [7].

In our previous work 11], numerical simulations on circular hydraulic jumps were conducted using some newly developed numerical schemes for multi-fluid flows. We investigated the transition from a type I jump to a type II jump. Non-hydrostatic pressure distributions in the gravitational direction were observed in our simulations. In our studies, we call 'dynamic pressure' the net amount of the pressure resulting from extracting the hydrostatic pressure from the actual pressure. We found that the dynamic pressure around the jump, which has been neglected in most of the theoretical studies to date, is important for the transition. In a type I jump, a steeper jump is always associated with a higher wall height ( [7] and Fig. 3). Thus, as $d$ is increased, the curvature of the interface immediately after the jump becomes larger, then the surface tension is strengthened, because the surface tension is proportional to the curvature. In order to counteract this surface tension and keep the jump surface steady, a larger rise in pressure is required (Figs. A $(a, b))$. If the wall height is increased over the critical $d_{c}$, the reverse pressure gradient generated by the dynamic pressure becomes stronger than the flow from below and a transition occurs.

In this Rapid Communication, we intend to clarify the relationship between the roller and the pressure field. The simulation results show that the single high dynamic pressure region in a type I jump becomes two regions after the transition to a type II jump. These two high pressure regions are located along the jump slope around the outer edge $R_{\text {out }}$ and the inner edge $R_{\text {in }}$ of the roller. This pressure distribution appears important to the flow separation at the outer edge of the roller and then essential to the maintenance of a roller.

The governing equations, including effects of gravity, viscosity and surface tension can be written as

$$
\frac{\partial \rho}{\partial t}+(\mathbf{u} \cdot \nabla) \rho=-\rho \nabla \cdot \mathbf{u}
$$




$$
\begin{gathered}
\frac{\partial \mathbf{u}}{\partial t}+(\mathbf{u} \cdot \nabla) \mathbf{u}=-\frac{\nabla p}{\rho}+\mathbf{g}+\frac{\mu}{\rho} \Delta \mathbf{u}+\frac{\mathbf{F}_{\mathbf{s v}}}{\rho} \\
\frac{\partial e}{\partial t}+(\mathbf{u} \cdot \nabla) e=-\frac{p}{\rho} \nabla \cdot \mathbf{u}
\end{gathered}
$$

where $\rho$ is the density, $\mathbf{u}$ the velocity, $\mathrm{p}$ the pressure, $\mathbf{g}$ the gravitational acceleration, $\mu$ the viscosity coefficient, $\mathbf{F}_{\mathbf{s v}}$ the surface tension force, and $e$ the inner energy. Both the liquid and the gas are assumed to have an equation of state in the form of a polytropic gas, but with quite different sound speeds (large for the liquid phase).

The numerical model is constructed based on the CCUP (CIP-Combined, Unified Procedure) method [12, the level set method 13, 14 and the CSF (Continuum Surface Force) model 15. By using the C-CUP method to solve multi-fluid flows, we are able to deal with both the gas and the liquid phase in a unified framework, and explicit treatment of the free boundary and interfacial discontinuity is not needed.

The interface between the liquid and the gas is tracked using the level set method with the CIP (Cubic Interpolated Propagation) method [16] as the advection solver. A density function $\phi$ generated from the level set function of the level set method by the Heaviside function can be set as $\phi=1$ for the liquid and $\phi=0$ for the air. The density function is then used to define the physical properties, such as sound speed and viscosity for different materials.

The surface tension force is modeled as a body force $\mathbf{F}_{\mathbf{s v}}$ calculated by the gradient of the density function, $\mathbf{F}_{\mathbf{s v}}=\sigma \kappa \nabla \phi$, where $\sigma$ is the fluid surface tension coefficient and $\kappa$ the local mean curvature. $\kappa$ is computed from $\kappa=-(\nabla \cdot \mathbf{n})$, where $\mathbf{n}$ is the outgoing unit normal vector to the interface and is evaluated from the level set function [14].

An axis-symmetric model has been constructed to deal with the circular hydraulic jump. The configuration of the simulation model on an $\mathrm{r}-\mathrm{z}$ plane is shown in Fig. 2. This calculation model is validated by comparing the computed results with the scaling relation [6,11.

Simulations were carried out with different heights of the outer circular wall $d$. The volume flux of the inflow is $Q=5.6 \mathrm{ml} / \mathrm{s}$ and the viscosity of the liquid is $\nu_{l}=7.6 \times$ $10^{-6} \mathrm{~m}^{2} / \mathrm{s}$. The steady surface profiles for the various wall heights are shown in Fig. 3. The three lower profiles are type I jumps, and the two upper profiles are type II jumps. We observe that the jump becomes steeper as the wall height increases for a type I jump, while for a type II, the slope of the jump appears less steep than that of type I with a high wall hight. These are consistent with the experimental results [7]. The roller is usually a consequence of a steepened jump, while its occurrence always leads to the destruction of the steepness.

The dynamic pressure distributions of the second, the third and the fourth profiles from the lowest were plotted in Figs. 14(a-c). For the cases of type I (Figs. 14(a,b)), a high pressure region (referred to hereafter as the primary high pressure) dominating a wide region under the jump surface is observed. In a type II jump, two high pressure regions are developed around the inner side of the jump (referred to as the primary high pressure) and the outer side of the jump (refereed to as the secondary high pressure) as shown in Fig. 4(c). This pressure distribution is essential for the roller. We observe that the high pressure on the outer side of the jump (the secondary high pressure) coincides with the separation point of the flow, as shown in Fig. A(d). This secondary high pressure continuously provides a pressure gradient force to maintain the upper reverse flow for the roller. The secondary high pressure is associated with the surface tension. In the steady state of the type II, the liquid surface appears convexly curved around the secondary high pressure region or the outer edge of the roller (Figs. $1(\mathrm{c}, \mathrm{d})$ ). This feature of the free surface around the outer edge of the roller is also observed in experiment [7]. To counteract the surface tension caused by this curved surface the small curvature and keep a steady surface, the secondary high pressure must be required. The reverse flow from the separation point moves down along the jump surface until it meets another high pressure (the primary high pressure) on the upstream side of the jump. The fluid motion is decelerated when it approaches the high pressure on the inner side of the jump. The direction of motion is then changed, and joins the main stream again around the confluent point $R_{i n}$.

We further simulated the disappearance process of a roller (the transition process from a type II jump to a type I jump) to study the details of the relation between the pressure field and the roller. We started from the steady state of a type II jump (the fourth profile from the lowest in Fig. 3). Its surface profile is shown as the topmost one in Fig. 5. The time of this initial state was set $t=0$. We simulated until the steady state of a type I jump (the second profile in Fig. 3) was reached by lowing the wall height at $t=0$. Fig. 5 displays the surface profiles at different instants. The flow experienced a transition from a type II jump to a type I jump. The evolution of the dynamic pressure field and the maximum value of the secondary high pressure are shown in Fig. 6. The initial pressure distribution is characterized by two high pressure regions and a roller as discussed above. As time increases, the secondary high pressure becomes weaker, and finally vanishes around $0.55 \mathrm{~s}$. It appears that the reduction of the secondary high pressure is associated with the decline in the curvature of the surface around the secondary high pressure. Meanwhile, the primary high pressure does not experience any significant change and finally becomes to the primary high pressure in the type I jump. In order to give a quantitative measure for the roller, we calculated the horizontal width of the roller as $R_{\text {out }}^{r}-R_{\text {in }}^{r}$. Fig. 7 shows the time evolution of the roller width $\left(R_{\text {out }}^{r}-R_{\text {in }}^{r}\right)$. With the secondary high pressure abating, the roller width decreased. This process was significantly enhanced after the secondary high pressure disappeared completely (from $0.55 \mathrm{~s}$ ) because the pres- 
sure gradient becomes perfectly opposite to the reverse flow of the roller. Around $0.75 \mathrm{~s}$, the roller disappeared. With the secondary high pressure and the roller having abated, the fluid eventually approached the steady state of a type I jump.

From this study, we have made clear that the existence of the high dynamic pressure regions and a secondary high pressure region around the outer edge of a roller are essential to the maintenance of a roller. The establishment of the high pressure field is a result of the balance among various fluid stresses, and the surface tension appears to play an important role. The secondary high pressure provides a driving force to generate a reverse current beneath the jump surface in a type II jump.

We would like to thank S. Watanabe and K. Hansen for many discussions. We also acknowledge the support of C. W. Stern. Numerical computations for this work were partially carried out at the Computer Information Center, RIKEN and the Yukawa Institute for Theoretical Physics, Kyoto University.

[1] Lord Rayleigh, Proc. Roy. Soc. London A 90, 324 (1914).

[2] I. Tani, J. Phys. Soc. Jpn. 4, 212 (1949).

[3] V.T. Chow, Open channel hydraulic, McGraw-Hill, New York, 1959.

[4] E. J. Watson J. Fluid. Mech. 20, 481 (1964).

[5] A.D.D. Craik et al., J. Fluid Mech. 112, 347 (1981).

[6] T. Bohr, P. Dimon, and V. Putkaradze, J. Fluid. Mech. 254, 635 (1993).

[7] T. Bohr et al., Physica B 228, 1 (1996).

[8] T. Bohr, V. Putkaradze, and S. Watanabe, Phys. Rev. Lett. 79, 1038 (1997).

[9] C. Ellegaard et al., Nature 392, 767 (1998).

[10] C. Ellegaard et al., Nonlinearity 12, 1 (1999).

[11] K. Yokoi and F. Xiao, Phys. Lett. A 257, 153 (1999).

[12] T. Yabe and P.Y. Wang, J. Phys. Soc. Jpn. 60, 2105 (1991).

[13] S. Osher and J.A. Sethian, J. Comput. Phys. 79, 12 (1988).

[14] M. Sussman, P. Smereka, and S. Osher, J. Comput. Phys. 114, 146 (1994).

[15] J.U. Brackbill, D.B. Kothe, and C. Zemach, J. Comput. Phys. 100, 335 (1992).

[16] T. Yabe et al., Comput. Phys. Commun. 66, 233 (1991).

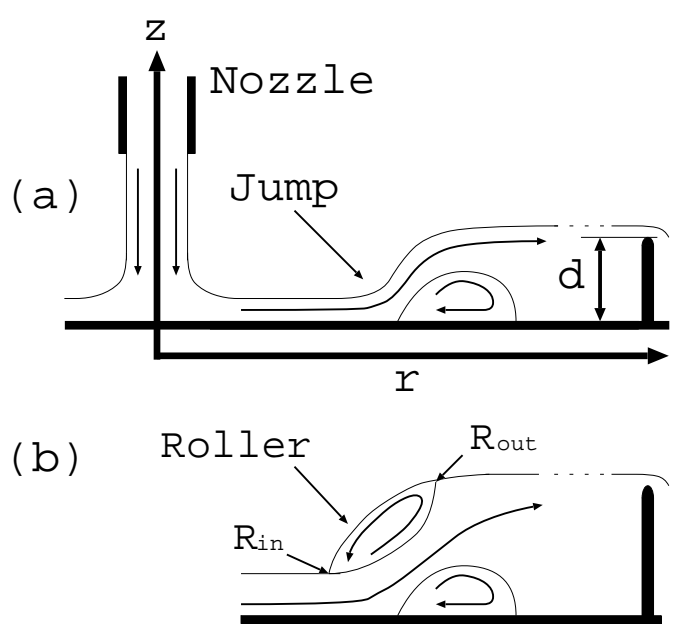

FIG. 1. Schematic figures of the circular hydraulic jump. The radius of the wall is much larger than the radius of the jump. The flow from the nozzle is constant. In this experiment, a high viscous liquid is used for controlling the instability of flow pattern. (a) and (b) are called type I and type II, respectively. The points of the inside and outside of the roller are defined as $\left(R_{\text {in }}^{r}, R_{\text {in }}^{z}\right)$ and $\left(R_{\text {out }}^{r}, R_{\text {out }}^{z}\right)$.

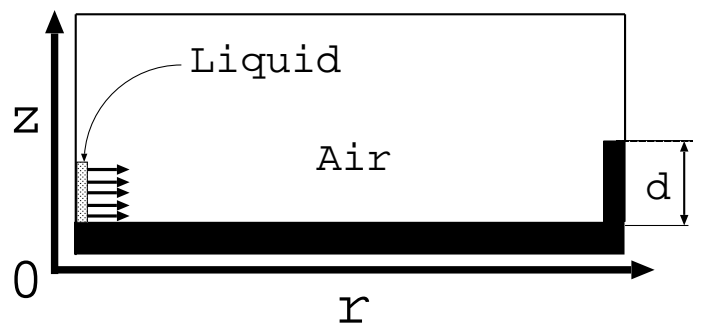

FIG. 2. Schematic figure for the initial condition of the simulation. The dark part indicates the no-slip wall. The liquid is jetted from the lower left to the right direction. A Cartesian grid with $\Delta r=\Delta z=0.1 \mathrm{~mm}$ is used.

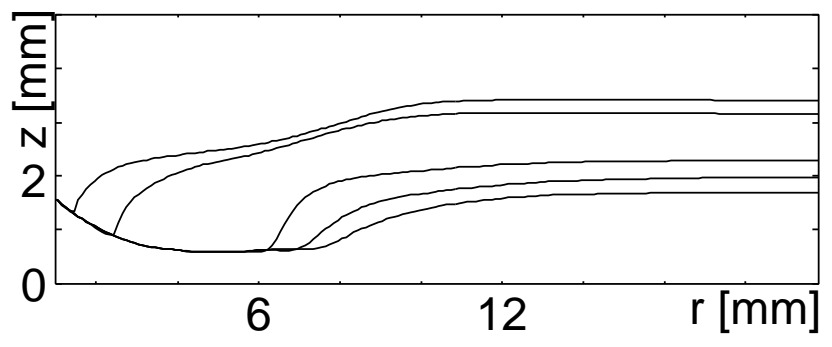

FIG. 3. Surface profiles for varying wall heights. $Q=5.6$ $\mathrm{ml} / \mathrm{s}$ and $\nu_{l}=7.6 \times 10^{-6} \mathrm{~m}^{2} / \mathrm{s}$ are used. The maximum values of the dynamic pressure around the jump (the primary high pressure) in type I jumps were $1.77,3.99$, and $8.47 \mathrm{~Pa}$ from the lowest respectively. 


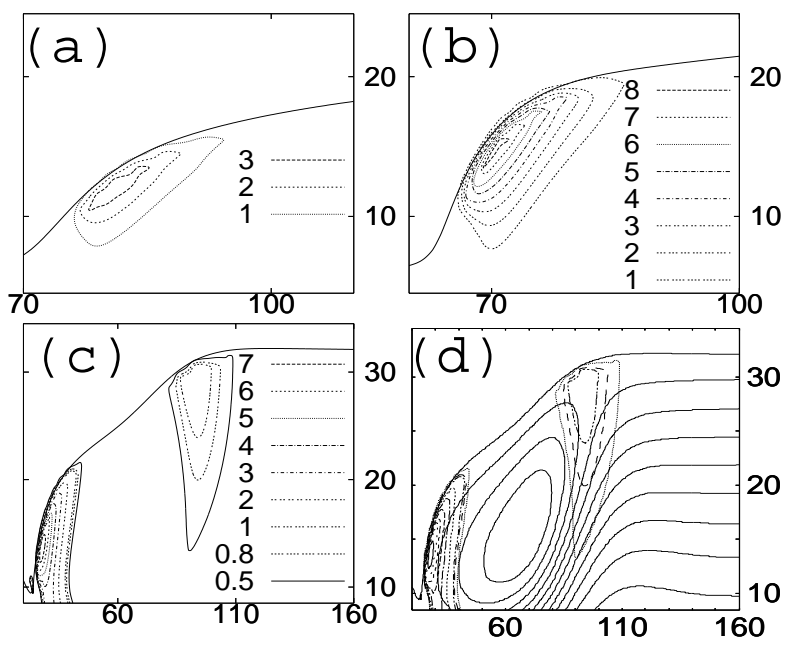

FIG. 4. Dynamic pressure $(\mathrm{Pa})$ contours and the surface profiles around the jump of the second (a), the third (b), and the fourth (c) from the lowest in Fig. 3. (d) shows the streamline, the dynamic pressure contours, and the surface profile of the fourth profile.

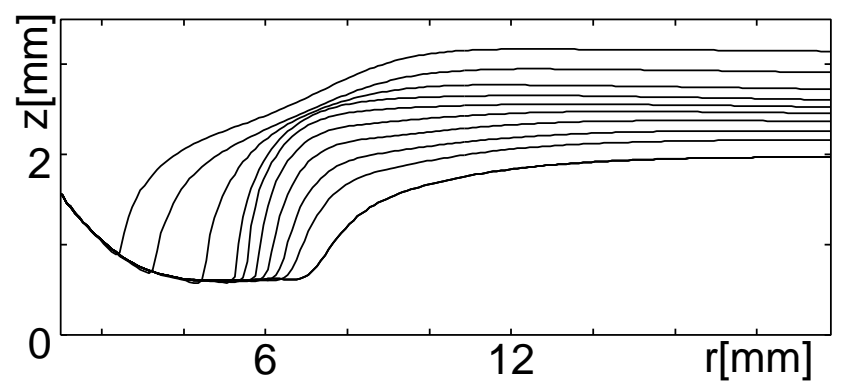

FIG. 5. Time evolution of the surface profile from the type II jump to the type I jump at $0.294 \mathrm{~s}$ intervals. The topmost profile is the initial state.

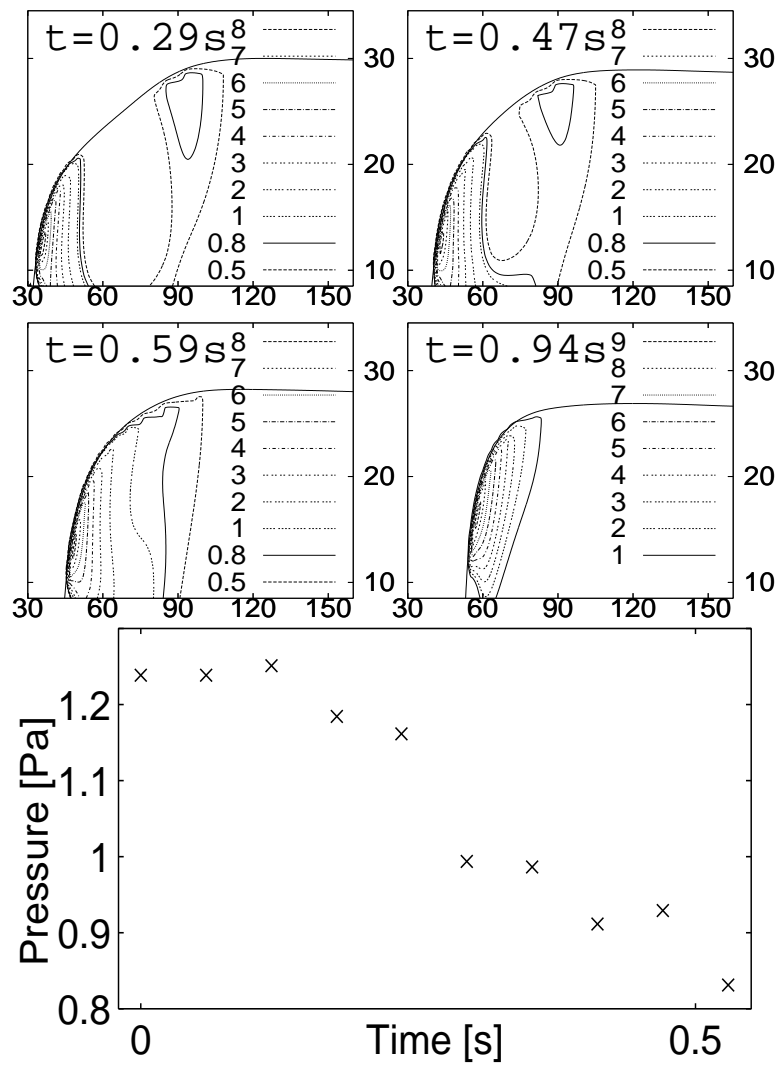

FIG. 6. Time evolution of the dynamic pressure distribution and the maximum value of the secondary high pressure. Fig. (c) and (a) correspond to $t=0$ and the final steady state.

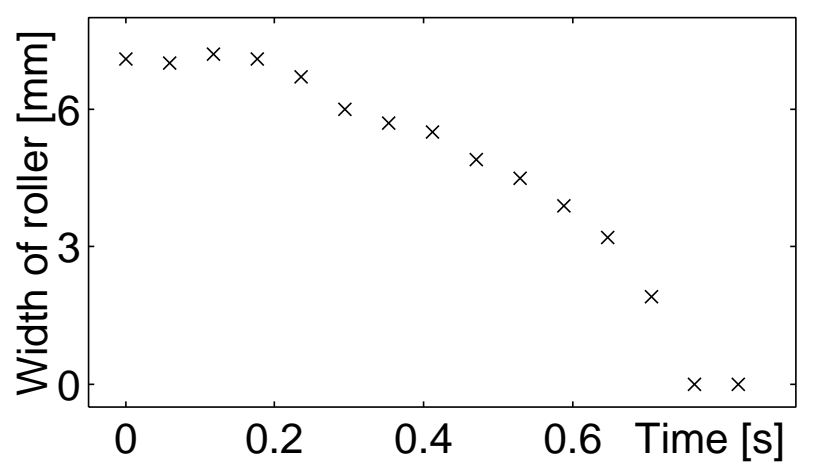

FIG. 7. Time evolution of the width of the roller. 